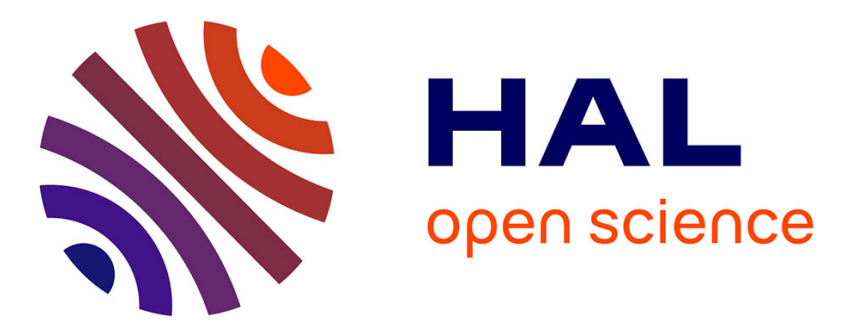

\title{
Intérêts et limites des suivis de cohorte pour comprendre les situations de handicap de l'enfant
}

Mélissa Arneton, Amélie Courtinat-Camps, Bertrand Geay, Corinne Bois

\section{To cite this version:}

Mélissa Arneton, Amélie Courtinat-Camps, Bertrand Geay, Corinne Bois. Intérêts et limites des suivis de cohorte pour comprendre les situations de handicap de l'enfant. Spirale - Revue de Recherches en Éducation , 2016. hal-01523546

\section{HAL Id: hal-01523546 \\ https://hal.science/hal-01523546}

Submitted on 2 Oct 2017

HAL is a multi-disciplinary open access archive for the deposit and dissemination of scientific research documents, whether they are published or not. The documents may come from teaching and research institutions in France or abroad, or from public or private research centers.
L'archive ouverte pluridisciplinaire HAL, est destinée au dépôt et à la diffusion de documents scientifiques de niveau recherche, publiés ou non, émanant des établissements d'enseignement et de recherche français ou étrangers, des laboratoires publics ou privés. 


\section{Version auteurs de l'article}

Arneton, M., Courtinat-Camps, A., Geay, B. \& Bois, C. (2016). Intérêts et limites des suivis de cohorte pour comprendre les situations de handicap de l'enfant. Spirale-Revue de recherches en éducation, 57 [numéro thématique «Petite enfance et politique inclusive : quelle prise en compte du handicap ?»], 109-119.

\section{Auteur 1, auteur correspondant}

Mélissa Arneton

Institution de rattachement : Grhapes EA 7287/ INS HEA

Adresse de correspondance : 58/60 avenue des landes 92150 Suresnes

Mail : melissa.arneton@inshea.fr

\section{Auteur 2}

Amélie Courtinat-Camps

Institution de rattachement : EA 1697 PDPS - Université de Toulouse, France

Mail : courtina@univ-tlse2.fr

\section{$\underline{\text { Auteur } 3}$}

Bertrand Geay

Institution de rattachement : CURAPP-ESS (UMR CNRS 7319) - Université de Picardie,

France

Mail : bertrand.geay@u-picardie.fr

\section{$\underline{\text { Auteur } 4}$}

Corinne Bois

Institution de rattachement : Conseil Départemental des Hauts-de-Seine, service départemental de PMI (Nanterre, France) 
Unité mixte Elfe (Ined- Inserm- EFS), Ined (Paris, France)

Mail : corinne.bois@ined.fr 


\title{
Titre
}

Intérêts et limites des suivis de cohorte pour comprendre les situations de handicap de l'enfant

\section{Résumé :}

L'évolution des compréhensions du handicap dans une perspective situationnelle et relationnelle, et l'apparition du concept «besoins éducatifs particuliers »(BEP), incitent à prendre en compte des besoins individuels de chaque enfant et ce, dès la prime enfance. Dans cette perspective, les bases de données quantitatives construites avec un recueil longitudinal sont une opportunité pour étudier et comprendre les liens entre le contexte familial, les éléments socio-économiques et les expériences vécues. Elles permettent de considérer les BEP et/ou les situations de handicap, dans une perspective vie entière, dès leur apparition, ou dès leur prise en compte, dans le parcours de l'enfant. Notre article se propose de discuter les intérêts et limites de telles bases pour aborder la petite enfance de ces enfants. Deux cohortes pluridisciplinaires de grande envergure sont présentées : la Millennium Cohort Study et la National Educational Panel Study. Deux cohortes françaises sont décrites plus précisément: ELFE et Epipage-2. La conclusion met en perspective les critiques fréquemment adressées aux cohortes avec leur pertinence pour étudier les enfants en situation de handicap.

Mots-clés : aménagement, approche longitudinale, besoins éducatifs particuliers, cohorte, éducation inclusive, enfance, situation de handicap.

\begin{abstract}
:
Developments in situational and relational perspective of disability and the emergence of the concept "special educational needs" incite taking into account the individual needs of each child since early childhood. To study and to understand the relationship between family background, socioeconomic factors and experiences, quantitative databases built in a longitudinal
\end{abstract}


perspective are an opportunity. They allow considering the BEP and / or handicap situations, in a life span perspective, when they occur or when they are taken into account in the child's path. Our paper attempts to discuss the interests and the limits to use such bases to study the primary childhood of these children. Two extensive and pluridisciplinary cohorts are presented: the Millennium Cohort Study and the National Educational Panel Study. Two French cohorts are described specifically: ELFE and Epipage-2. The conclusion put in perspectives the frequent critics addressed to cohorts with their utility to study the children in situation of disability.

Key words: special educational provision, longitudinal approach, special educational needs, cohort, inclusive education, childhood, situation of disability. 


\section{Introduction}

Si les recherches sont nombreuses à porter sur les adultes en situation de handicap quand elles sont produites dans le cadre de grandes enquêtes (inter)nationales, rares sont celles menées sur la petite enfance. Notre article se propose de présenter quelques bases de données quantitatives concernant la petite enfance et pouvant faire l'objet d'analyses secondaires. Les études et les panels internationaux se réfèrent à la définition de la classification internationale du fonctionnement du handicap et de la santé (CIF). Elles conçoivent le handicap comme toute limitation d'activité ou restriction de participation à la vie en société subie dans son environnement par une personne en raison d'une altération substantielle, durable ou définitive d'une ou plusieurs fonctions physiques, sensorielles, mentales, cognitives ou psychiques, d'un polyhandicap ou d'un trouble de santé invalidant.

S'inspirant d'une approche sociale, ou approche « situationnelle » du handicap, et sans négliger les déficiences développées dans le modèle biomédical, la CIF met principalement l'accent sur les facteurs environnementaux dans la production des situations de handicap. Nous inscrivons notre propos dans cette approche et dans le contexte d'apparition progressive du concept «besoins éducatifs particuliers» (BEP) (special educational needs). Cette nouvelle « catégorie » permet une distanciation au modèle médical, en le remplaçant par une approche dans laquelle les besoins individuels de chaque enfant doivent être identifiés et appréhendés, et ce dès la prime enfance. Cette notion, qui autorise à dépasser l'aspect administratif du handicap, met l'accent sur les éléments du contexte. Les notions de BEP et de handicap sont proches sans être pour autant superposables. En effet, la définition anglo-saxonne indique que les BEP peuvent être liés aussi bien à des difficultés graves d'apprentissage dont les origines n'ont pas été déterminées comme « organiques » qu'à un fonctionnement cognitif différent. Les individus à BEP constituent ainsi une population plus large que les seuls individus ayant une reconnaissance administrative du handicap. Les enfants ont un besoin particulier dès qu'une 
difficulté d'apprentissage requiert la mise en place d'aménagement spécifique (Special Educational Needs 2001). L'utilisation d'une même étiquette « élèves à BEP » pour tous ces profils suggère une analyse des éléments communs aux expériences individuelles des élèves reconnus comme éloignés de la norme éducative.

Le recours à des bases de données quantitatives peut permettre d'identifier des ensembles communs aux populations d'enfants à BEP et/ou en situation de handicap. À cette fin, la comparaison des données issues de bases concernant exclusivement des populations en situation de handicap à celles de populations tout venant est attendue. Les recueils de données permettant de constituer des bases nécessitent des autorisations de la part de l'état (commission nationale informatique et libertés en France par exemple), du comité de pilotage du dispositif d'enquête et des participants (familles et enfants quand ils sont en âge de donner leur avis). L'anonymat des participants est une exigence éthique incontournable. Plusieurs traitements, gérés par un système informatique de protection des données découplés en actions distinctes, sont faits avant que les données puissent être proposées à des fins de recherche. Aucun intervenant ne peut avoir accès à la fois à l'identité d'un enfant et aux données le concernant. Les demandes d'accès aux données répondent à un protocole de suivi administratif incluant la description du projet scientifique nécessitant l'accès aux données et des documents de contractualisation. Dans le cadre de l'analyse de trajectoires de vie des enfants, si des variables demandées permettent d'identifier des familles, elles ne seront pas mises à disposition. Le chercheur désireux de mettre en œuvre des analyses secondaires de données se doit donc de réfléchir à sa méthodologie de manière anticipée.

\section{Positionnement conceptuel}

L'article insiste sur l'intérêt de recourir à des suivis longitudinaux de cohortes pour considérer les BEP et/ou les situations de handicap, dans une perspective de vie entière (i:e dès leur apparition et prise en compte dans le parcours de vie des enfants). L'approche biographique 
défendue dans ces travaux (Larroque et alii 2011 ; Pirus et alii 2010) apporte des éléments essentiels à la compréhension du contexte familial, socio-économique, de son évolution, et des expériences vécues par l'enfant tout au long de son parcours (Elder 1998). Contrairement à une logique rétrospective, où le chercheur reconstruit des parcours de vie institutionnels et scolaires a posteriori, en mobilisant les parents et les individus concernés à la fin du suivi longitudinal, cette approche recueille les données au fur et à mesure.

Les travaux internationaux portant sur les enfants tout-venant, qui incluent de fait des enfants en situation de handicap, comme la Millennium Cohort Study, la NEPS (National Educational Panel Study) ou ELFE (Etude longitudinale française depuis l'enfance), permettent d'identifier les mécanismes visibles et invisibles de sélection (Dubet, Duru-Bellat et Vérétout 2010 ; Merle 2012) et de désignation des élèves à BEP (OCDE 2000). Nous pensons que ce processus de désignation peut être mis en lumière, notamment en notant les aménagements mis en œuvre tout au long de la vie de l'enfant par les institutions et les familles. Ces aménagements sont dépendants des politiques publiques mises en œuvre nationalement. Compte tenu des spécificités du modèle social français en termes de services dédiés à la petite enfance, et l'existence d'une possibilité de scolarisation dès 3 ans, nous présentons deux cohortes françaises dont la démarche et les indicateurs aident à mieux appréhender la situation française. Une étude menée avec ELFE en partenariat avec les médecins de protection maternelle infantile (PMI), et une étude qualitative menée auprès de 36 familles (VenirOMonde) enrichissent les données de la cohorte Elfe. Dans la conclusion, nous mettons en débat les critiques fréquemment adressées aux études de cohortes quant à leur pertinence dans l'étude des enfants en situation de handicap.

\section{Handicap et BEP dans des bases de données relatives à la petite enfance}

Par la diversité des méthodes d'échantillonnage, du suivi des participants et des modes de collecte, les cohortes d'enfants sont riches d'enseignements et sont des outils incontournables 
pour les recherches sur le développement de l'enfant aussi bien dans une perspective épidémiologique (Spira 2013) qu'en sciences sociales (Pirus et alii 2010) ou en sciences humaines (Pulkkinen et Kokko 2012).

Lorsque que ces études sont conduites par des acteurs de la statistique publique ${ }^{1}$, les données sont récoltées auprès de larges échantillons représentatifs de la population, ce qui permet de généraliser les résultats obtenus et d'augmenter l'utilité sociale de ce type d'étude. Les analyses secondaires des données recueillies par cohorte sont un moyen d'obtenir des données relatives à des sous-populations (dans notre cas, des enfants en situation de handicap et à BEP) pour lesquelles il n'existe pas de cadre d'échantillonnage évident. Les cohortes d'envergure constituent une méthode pour mesurer l'impact d'un nouveau dispositif d'action sociale ou la mise en œuvre d'une nouvelle politique publique. La constitution de sous-échantillons autorise des analyses comparatives pour identifier par exemple les relations entre les prises en charge effectuées dans un pays et les représentations du handicap. Les résultats peuvent contribuer à l'élaboration de préconisations relatives aux pratiques ou à orienter les politiques publiques concernant l'accès des personnes en situation de handicap à leurs droits (NESSE 2012). Ces analyses permettent de veiller à l'application de la convention des Nations-Unies relative aux droits des personnes handicapées pour une participation pleine et entière des personnes en situation de handicap (Crawford, Dinca-Panaitsecu, Fougeyrollas et Rioux 2012; Madans, Loeb et Altman 2011). D'autres bases sont utiles pour étudier les conditions du développement des sujets en situation de handicap notamment les projets pluridisciplinaires de cohortes d'enfants suivis de la naissance (ou dès leurs premières années) à l'âge adulte. A partir de données recueillies tout au long de la vie de l'enfant, les cohortes autorisent l'étude a posteriori de l'influence des aménagements et des soutiens rapportés par les parents sur les socialisations

\footnotetext{
${ }^{1}$ Ils assurent également un archivage pérenne et un suivi du respect des droits des participants.
} 
éducatives. Selon nous, elles peuvent contribuer à répondre à certaines interrogations dont : Quelles sont les étapes de désignation conduisant à la mise en place d'un aménagement ? Quelles sont les différences d'aménagement entre institutions et familles et entre les institutions ? Peut-on déceler des effets différenciés de ces aménagements sur l'enfant aux niveaux cognitif, affectif et social et ce, plus particulièrement, pour ceux qui sont, ou seront, considérés à BEP ? Etudier les aménagements est un moyen pour mettre en évidence la divergence d'itinéraires préscolaires puis scolaires, et voire si l'hétérogénéité des parcours induit une hétérogénéité dans les manières dont ces enfants «prennent part » à la société.

De quelles données les chercheurs peuvent-ils se saisir? Plusieurs grandes enquêtes sont référencées à l'étranger et depuis peu en France (Pirus et Leridon 2010). Nous nous attachons plus précisément à deux d'entre elles (la Millennium Cohort Study et la National Educational Panel Study), et ce, même si d'autres cohortes permettent d'étudier des éléments de parcours des enfants présentant un BEP. Par exemple, l'étude longitudinale du développement des enfants du Québec (ELDEQ) a permis notamment de mieux comprendre l'apparition des signes d'hyperactivité-impulsivité et d'inattention chez les enfants (Cardin, Desrosiers, Belleau, Giguère et Boivin 2011). Quant à l'étude longitudinale australienne, elle a contribué à favoriser la formation à la prise en charge des BEP des éducateurs de jeunes enfants (Williamson, Davis, Priest et Harrison 2011).

Présentons tout d'abord, la cohorte britannique, Millennium Cohort Study ou MCS, l'héritière d'une longue tradition d'études menées de manière longitudinale. Cette cohorte spécifie les éléments concernant les handicaps, les BEP et les aménagements dont bénéficient les enfants durant leur vie à l'école et en dehors de l'école. Elle concerne 18819 enfants (18 553 familles) nés en 2000 et 2001. Les thématiques abordées concernent l'impact de la prématurité sur le développement psychomoteur de l'enfant (capacités cognitives et socialisation), l'étude de la parentalité et de l'implication des parents dans l'éducation des enfants, le rôle de 
l'environnement social dans le développement de l'enfant (réseau social, familial) mais aussi des thématiques de santé (allergies et maladies développées par l'enfant, rôle de l'allaitement, accès aux services de santé). Ces données permettent aussi bien de mener des études généralistes sur le handicap chez les jeunes enfants (Parsons et Platt 2013) que de circonscrire une population spécifique comme les enfants déficients visuels (Harris, Keil, Lord et McManus 2012).

Evoquons ensuite, la National Educational Panel Study (NEPS) conduite en Allemagne depuis 2009. Sa particularité est d'avoir été conçue dès l'origine en prenant en compte des questions méthodologiques à propos des enfants ayant des BEP. Il s'agit d'une étude longitudinale plus large que la MCS puisqu'elle est constituée de plusieurs cohortes représentatives d'enfants et adolescents ainsi que d'une cohorte d'adultes (soit un total de 60000 participants). En 2009, une première cohorte représentative est constituée d'enfants âgés de quatre ans scolarisés en Kindergarten. Depuis 2012 les enfants âgés entre un et quatre ans et ceux de six ans sont intégrés au dispositif longitudinal de suivi. Cette enquête autorise à la fois une analyse longitudinale des données afin d'étudier les acquisitions et le développement des compétences au cours de la vie d'une classe d'âge, et une analyse transversale, en tenant compte des effets de changements de politiques concernant l'enfance ou l'éducation (Blossfeld, Schneider et Doll 2009). L'intérêt de cette cohorte pour l'étude des BEP concerne la prise en compte de l'hétérogénéité de cette catégorie. Les coordinateurs ont choisi de suivre une cohorte spécifique composée d'enfants en difficulté d'apprentissages en plus des enfants identifiés comme étant à BEP intégrés au sein des 60000 participants par hasard (Heydrich, Weinert, Nusser, Artelt et Cartensen 2013). Ce choix méthodologique résulte d'une part des caractéristiques du système scolaire allemand, dans lequel de nombreux enfants à BEP sont scolarisés en milieu spécialisé, et d'autre part du fait que plus de $40 \%$ des enfants allemands ayant un BEP sont identifiés comme ayant des difficultés d'apprentissage. 
Si ces cohortes ne permettent pas d'appréhender directement les effets d'un paradigme d'inclusion sur les situations des enfants ou sur les relations qu'entretiennent les parents et les acteurs professionnels, elles fournissent néanmoins des éléments sur les conditions du développement des enfants. La mobilisation dans les différentes cohortes de catégories comparables favorise l'investigation de questions relatives à l'inclusion dans différents pays.

\section{Centration sur des données nationales : le cas français}

Rappelons tout d'abord quelques éléments concernant les services dédiés à la petite enfance en France avec notamment l'existence d'une possibilité de scolarisation dès 3 ans (Florin 2007 ; Rayna et Plaisance 1997). L'accueil des 0-3 ans dépend du ministère des affaires sociales et de la santé (aussi bien pour l'accueil individualisé auprès d'une assistante maternelle que pour l'accueil en collectivité comme dans une crèche) tandis que celui des 3-6 ans dépend du ministère de l'éducation nationale, et ce, même si l'école n'est pas obligatoire avant 6 ans. L'accueil des enfants en situation de handicap dans les différents lieux de vie relève du droit commun $^{2}$. En effet, l'inclusion des enfants en situation de handicap ou à BEP est désormais reconnue comme la voie la plus adaptée sur le plan international comme en France pour prévenir l'exclusion et garantir à tous une éducation de qualité (ONU 2006 ; UNESCO 1994).

Les informations relatives aux enfants en situation de handicap avant l'âge de trois ans sont très rares (Candiago, Moreira, Ruffiot, Robin et Maneveau 2012). Si la scolarisation des enfants de 3 ans et moins représente $13,55 \%$ des enfants tout-venant scolarisés en maternelle et si celle des 3-6 ans est majoritaire (Villaume et Legendre 2014), ce n'est pas le cas pour les enfants en situation de handicap ou à BEP. À la rentrée 2013, sur les 141565 élèves identifiés comme étant en situation de handicap, 1,9\% est âgé de 3 ans et moins, 4,7\% de 4 ans, 7,7\% de 5 ans et 10,4\% de 6 ans (Moisan 2014). Bien qu'intéressantes pour établir des états des lieux, ces

\footnotetext{
${ }^{2}$ Décret du 1er août 2000 modifié en 2007 et 2010, loi du 2 janvier 2002, loi du 11 février 2005, convention d'objectif et de gestion 2009-2012, décret du 2 avril 2009.
} 
enquêtes ne permettent pas d'étudier le développement de l'enfant dans sa globalité. Or la période 0-6 ans concentre des moments décisifs de la prise de conscience des besoins particuliers de l'enfant, du diagnostic et de l'aménagement de l'environnement familial et éducatif. Cette phase déterminante pour l'élaboration du projet de vie et la future scolarisation est étroitement liée aux modalités d'accueil en milieu ordinaire. C'est aussi à ce stade que vont s'établir les actions coordonnées entre approches éducative, médicale et médico-sociale. Il est nécessaire d'analyser l'inclusion d'un point de vue systémique, depuis les principes généraux (sociaux, juridiques et moraux) jusqu'aux interactions éducatives et scolaires entre les professionnels et les enfants, en passant par le rôle déterminant des établissements et des partenariats (Ebersold 2009).

Deux cohortes françaises concernent des enfants depuis leur naissance ${ }^{3}$ : ELFE (Enquête longitudinale française de l'enfance), dans laquelle nous sommes impliqués, et EPIPAGE-2 (Etude épidémiologique sur les petits âges gestationnels). La première cohorte porte sur la population générale tandis que l'autre concerne une population spécifique. Le croisement des résultats issus de chacune d'elle permet de prendre en compte la diversité des situations de handicap : les situations de handicaps liées à la gestation ou apparaissant dès la naissance, aussi bien que les handicaps de développement ou les situations de développement atypique (difficultés d'apprentissage, haut potentiel intellectuel).

ELFE est la première étude longitudinale française consacrée au suivi des enfants, de la naissance à l'âge adulte ${ }^{4}$. Lancée auprès de 500 familles pilotes en 2007, elle est généralisée en France métropolitaine depuis 2011 et concerne plus de 18300 enfants. La gestion est assurée par une équipe mixte de suivi menée par l'institut national d'études démographiques, l'institut

\footnotetext{
${ }^{3}$ Les enfants sont âgés de trois ans et demi en 2015.

${ }^{4}$ L'une des premières cohortes généralistes françaises est l'étude sur les déterminants pré et post-natals précoces du développement psychomoteur et de la santé de l'enfant. Initiée en 2003, elle est à forte dominante en épidémiologie et en santé.
} 
national de la santé et de la recherche médicale et l'établissement français du sang. ELFE représente une occasion unique d'étudier sur un échantillon représentatif créé par grappes, les liens entre les situations de handicaps d'enfants nés à plus de 33 semaines d'aménorrhées et leur contexte de socialisation notamment de la naissance à 2 ans (recueil en cours de traitement). La cohorte EPIPAGE-2, débutée aussi en 2011, concerne 7804 enfants nés avant 33 semaines de durée d'aménorrhées et qui seront suivis jusqu'à l'âge de 12 ans. Cette étude nationale a pour but de mieux connaître le devenir des enfants prématurés (à l'exception des prématurités tardives). Le croisement des données ELFE avec les données issues d'EPIPAGE-2 permet d'étudier de manière contrastée si les BEP sont plus liés à des environnements ou événements de vie spécifiques ou à des conséquences d'une grande prématurité de naissance.

Dans les deux cohortes, deux types de variables permettent d'identifier des enfants en situation de handicap. Les premières sont administratives : un item porte sur le congé de présence parentale (qui est attribué lorsque l'enfant est en situation de longue maladie ou de handicap) et un autre peut être calculé à partir des plafonds des aides financières en tenant compte du complément lié à l'allocation journalière de présence parentale versée par les caisses d'allocations familiales. Les secondes variables identifient des situations de handicap à partir d'éléments diagnostiques concernant le développement psychomoteur ou affectif de l'enfant. En plus de ces indicateurs médicaux, une question relative à la perception par les parents de l'état de santé de leur enfant indique s'ils le considèrent comme étant en bonne ou en mauvaise santé. Enfin, dans ELFE une variable invite les parents à déclarer si un membre de la famille est en situation de handicap.

Contrairement à la NEPS, il n'est pas prévu de créer une cohorte spécifique relative à un BEP en particulier. L'intérêt majeur d'ELFE est, pour nous, de pouvoir étudier les mécanismes de désignation des catégories (à BEP ou en situation de handicap) qui vont s'appliquer (ou non) aux enfants. L'approche pluridisciplinaire mobilisée dans le suivi des enfants ELFE et 
EPIPAGE-2 est une opportunité pour développer les connaissances sur les socialisations primaires des enfants à BEP par rapport à celles des enfants qui n'en montrent pas. De plus, l'inscription des cohortes dans le cadre de la Life course approach ${ }^{5}$, permet d'observer l'impact de moments spécifiques (comme l'annonce du diagnostic par exemple) sur les trajectoires à venir de l'enfant et celles de sa famille. Les cohortes rendent possibles l'évaluation de la chronologie de ces différents événements afin de repérer les liens entre certaines pathologies à la naissance et le développement ultérieur des enfants (Grandjean 2008). Par ailleurs, le parcours des enfants en situation de handicap et de leur famille s'avère souvent complexe et parsemé de ruptures, du fait de la multitude des professionnels et des institutions auxquels familles et enfants sont confrontés. Dans ce contexte, étudier la façon dont se fédèrent les acteurs concernés s'avère nécessaire.

Le recours à des données longitudinales sur de grands échantillons facilite la mise en lumière des enjeux et des questionnements posés par le paradigme de l'inclusion. Si ELFE ou EPIPAGE-2 ne permettent pas directement d'interroger par exemple la manière dont les auxiliaires de puériculture et les éducatrices de jeunes enfants participent aux décisions liées à la prise en compte des besoins spécifiques de l'enfant « handicapé » (e.g. Bedoin et JannerRaimondi à paraitre), il est possible de mener des études spécifiques. Un partenariat de recherche a été ainsi initié par ELFE et les services départementaux de PMI en 2014-15, il se poursuit en 2015-2016. La prise en compte de l'enfant « différent » à l'âge de 4 ans dans le milieu de vie scolaire est approchée à partir du bilan de santé proposé par les PMI aux enfants scolarisés en maternelle, selon le fonctionnement spécifique à chaque département en petite ou moyenne section (Bois et Guillemot 2014). Réalisée dans 32 départements métropolitains, cette enquête permettra de documenter l'existence d'aménagements (projet d'accueil individualisé,

\footnotetext{
${ }^{5}$ Ce modèle développé ces vingt dernières années s'intéresse aux trajectoires individuelles dans une approche pluridisciplinaire.
} 
projet personnalisé de scolarisation), d'orientation ou de suivi (orthophoniste, centre d'action médico-sociale précoce...), d'anomalies du développement (psychomoteur, langagier) ou de déficiences sensorielles. L'enquête concerne les enfants nés les jours d'inclusion d'ELFE, dans des territoires très variés du point de vue géographique, de la population et de l'offre de soins. Il est prévu d'inclure plus de 10000 enfants dont un tiers appartenant à la cohorte ELFE. De la même façon, des recherches spécifiques en milieu scolaire sont envisagées ${ }^{6}$. En effet, les données issues de cohortes ou les études initiées à partir des enfants et des familles participantes peuvent permettre d'envisager d'autres manières de renforcer une coopération : facilitant le parcours des parents tout en améliorant le processus d'inclusion des enfants.

La mobilisation d'une méthodologie mixte de recueil et d'analyses des données est une autre possibilité offerte par les cohortes. Un exemple est le projet sur les primes socialisations des enfants à BEP partir de données ELFE et de VenirOMonde (versant qualitatif d'ELFE). Dans le volet quantitatif, les données issues des fichiers ELFE (maternité, 2 mois, 1 an) sont analysées afin de s'assurer que les taux de prévalence moyens observés au niveau national français se retrouvent bien dans l'échantillon des 18000 enfants participant à ELFE. Les caractéristiques des situations de handicaps ainsi que les caractéristiques socioéconomiques des familles sont étudiées au moyen d'analyses descriptives. Le volet qualitatif de la recherche permet, quant à lui, de fournir des éléments sur les parcours de vie des enfants en situation de handicap et de leurs familles. L'analyse des entretiens sociologiques menés aux mêmes étapes que pour ELFE permet de vérifier si les indicateurs présents dans les questionnaires favorisent l'accès aux spécificités des situations des familles dont un enfant est en situation de handicap et de réajuster, le cas échéant, les futurs protocoles

\footnotetext{
${ }^{6}$ Un protocole de recherche collaborative entre l'équipe ELFE et l'institut français de l'éduction a été lancé en 2014 concernant le suivi en école maternelle des enfants ELFE en 2015-2016 et 2016-2017.
} 
Mener ce type d'étude est intéressant pour analyser les aménagements proposés de manière globale aux enfants catégorisés «à BEP ». En Grande-Bretagne, Parsons et Platt (2013) ont comparé la pertinence de trois variables existantes dans la MCS pour identifier les enfants en situation de BEP : le retard développemental, une limitation de longue durée dans les activités en raison d'un problème de santé ou d'une maladie et la mention d'un ou plusieurs BEP. La prise en compte du niveau socio-économique des familles révèle des liens significatifs entre certains BEP (difficulté de comportement, troubles du langage) plutôt qu'avec d'autres comme la dyslexie. Etant donné les débats actuels sur la différenciation à apporter dans les aménagements proposés aux enfants à BEP ou sur la médicalisation de l'échec scolaire, il nous semble primordial mener des investigations longitudinales sur des données françaises concernant à la fois les situations de handicap et les BEP.

\section{Discussion}

Dans le cadre de la promotion d'une société inclusive, avoir plus d'informations sur l'environnement et les parcours des enfants en situation de handicap, ou plus généralement à $\mathrm{BEP}$, peut concourir à la mise en œuvre de politiques en proposant des préconisations (Maguire 2012) ou en facilitant l'évaluation scientifique de la pertinence d'une réponse politique par rapport à une autre (Parsons et Platt 2013). Dans cet article, nous avons souligné l'intérêt d'utiliser des cohortes pour éclairer sous un autre angle les problématiques concernant l'inclusion durant la petite enfance. Malgré tout, ces outils présentent des limites comme toutes bases de données (Block 1971 ; Pulkkinen et Kokko 2012 ; Ravaud 2014).

Selon nous, deux grandes catégories de limites se posent de manière saillante concernant l'usage des cohortes pour étudier les enfants à BEP et ou en situation de handicap. La première limite concerne la réduction et la simplification de la complexité des situations de vie. La seconde limite concerne l'approche pluridisciplinaire mobilisée dans les cohortes. Toutefois 
certaines caractéristiques des cohortes ou certains traitements statistiques qu'elles induisent, modèrent ces deux limites.

Si les cohortes peuvent réduire ou simplifier la complexité des situations de vie, le fait d'être constituées sur des échantillons appariés et randomisés est une occasion unique pour investiguer des questions dont l'éparpillement statistique limite la prise en compte de manière quantitative. De plus, les données recueillies sur de grands échantillons rendent possibles la mise en œuvre de méthodes d'analyses permettant de pallier certains écrasements des individus au profit des variables. Concernant la seconde limite relative à la mise en œuvre de protocoles pluridisciplinaires, il est important de noter que cette approche est un moyen de répondre à une limite majeure des suivis longitudinaux : la diminution du nombre d'enquêtés. Notamment, dans le cadre du suivi de sujets en nombre restreint, comme ceux en situation de handicap, éviter l'attrition est primordiale tout en conservant la richesse des facteurs. Prenons l'exemple de l'étude du développement psychomoteur de l'enfant. De nombreux facteurs interviennent dans son développement (moteur, cognitif, langagier, social et affectif notamment), ils relèvent tant du plan biologique (capacités neuronales, acuité visuelle ...) que du plan social (vie relationnelle, familiale, habitudes de vie...). Étudier séparément ces différents facteurs est certes intéressant mais plus réducteur. Une approche pluridisciplinaire favorise l'étude de leurs interactions, des suivis de cohortes menées de manière disciplinaire ne pourraient pas présenter une telle richesse. De plus, le travail pluridisciplinaire nécessite, de manière encore plus pressante, de rendre compte du positionnement conceptuel mis en œuvre dans les recueils afin de proposer des variables et des outils de mesure cohérents théoriquement et pouvant être utilisés à différentes étapes de vie du sujet; ce qui répond aussi partiellement à la première limite relative à la réduction de la complexité des situations de vie.

Bien que les cohortes présentent des limites théoriques et méthodologiques, les chercheurs s'accordent sur la pertinence de l'approche longitudinale pour étudier certaines questions qui 
ne pourraient pas l'être autrement. Dans le cadre d'une approche situationnelle du handicap, investiguer la permanence et le changement dans le parcours de vie d'un sujet est nécessaire, de même que comparer à différents âges de la vie les situations vécues par différents sujets.

\section{Bibliographie}

Bedoin D. \& Janner-Raimondi M. (dir.) (à paraître). Accueil de jeunes enfants en situation de handicap en crèche et maternelle. Grenoble : PUG.

Block J. (1971). Lives through time. Berkeley, CA: Bancroft Books.

Blossfeld H.P, Schneider T. \& Doll J. (2009) « Methodological advantages of panel studies. Designing the new National Educational Panel Study (NEPS) in Germany » - Journal for Educational Research Online 1 (10-32).

Bois C. \& Guillemot G. (2014). «Bilans de santé PMI 3-4 ans à l'école maternelle dans les Hauts-de-Seine, France : synthèse des études 2005 et 2010 et perspectives » - Bulletin épidémiologique hebdomadaire 29 (482-90).

Candiago P., Moreira M., Ruffiot A., Robin K. \& Maneveau R. (2012) «Les publics des établissements d'accueil du jeune enfant (EAJE). Attribution des places et gestion au quotidien »- Dossier d'études de la CAF 152.

Cardin J.-F., Desrosiers H., Belleau L., Giguère C. \& Boivin M. (2011) «Les symptômes d'hyperactivité et d'inattention chez les enfants de la période préscolaire à la deuxième année du primaire»-Portraits et trajectoires. Série Étude longitudinale du développement des enfants du Québec - ÉLDEQ 12.

Crawford C., Dinca-Panaitsecu M., Fougeyrollas P. \& Rioux P. (2012) « Towards a Statistical Model for Monitoring the Exercise of Human Rights under the UN Convention on the Rights of Persons with Disabilities - Canadian Case Study » - Review of Disability Studies 8, 4 (5769).

Dubet F., Duru-Bellat M. \& Vérétout A. (2010) Les Sociétés et leur école. Paris : Seuil. 
Ebersold S. (2009) « Autour du mot “inclusion"»-Recherches \& Formation 61 (71-83).

Elder G.H. (1998) « The life course as developmental theory »- Child Development 69, 1 (112).

Florin A. (2007) Modes d'accueil pour la petite enfance. Que dit la recherche internationale? Ramonville Saint-Agne : Érès.

Grandjean P. (2008) «Late insights into early origins of disease »-Basic and Clinical Pharmacology and Toxicology 102, 2 (94-99).

Harris J., Keil S., Lord C. \& McManus S. (2012) Sight impaired at age seven: Secondary analysis of the Millennium Cohort Study. Rapport remis à NatCen Social Research.

Heydrich J., Weinert S., Nusser L., Artelt C. \& Carstensen C.H. (2013) «Including students with special educational needs into large-scale assessments of competencies: Challenges and approaches within the German National Educational Panel Study (NEPS) »- Journal for Educational Research Online /Journal für Bildungsforschung Online 5 , 2 (217-240).

Larroque B., Ancel P.Y., Marchand-Martin L., Cambonie G., Fresson J., Pierrat V., Rozé J.C., Marpeau L., Thiriez G., Alberge C., Bréart G., Kaminski M., Marret S. \& Epipage Study group. (2011) «Special care and school difficulties in 8-year-old very preterm children: the Epipage cohort study »-PLoS One 6, 7.

Madans J. H., Loeb M.E. \& Altman B. M. (2011) « Measuring disability and monitoring the UN Convention on the Rights of Persons with Disabilities: the work of the Washington Group on Disability Statistics »- BMC Public Health 11 (1-8).

Merle P. (2012) La ségrégation scolaire. Paris : La Découverte.

Moisan C. (ed.) (2014) Repères et références statistiques sur les enseignements, la formation et la recherche. Edition 2014. Paris : Ministère de l'éducation nationale. 
NESSE network of experts (2012) Education and disability / special needs. Policies and practices in education, training and employment for students with disabilities and special educational needs in the EU. Rapport préparatoire pour l'Union européenne.

OCDE - Organisation de coopération et de développement économiques (2000) Besoins éducatifs particuliers : Statistiques et indicateurs. Paris : OCDE.

ONU - Organisation des Nations Unies (2006) Convention des Nations Unies relative aux droits des personnes handicapées (CDPH). New-York : ONU.

UNESCO - Organisation des Nations Unies pour l'éducation, la science et la culture (1994) Déclaration de Salamanque et analyse. Paris : UNESCO.

Parsons S. \& Platt L. (2013) «Disability among young children. Prevalence, heterogeneity and socio-economic disadvantages »- Centre for Longitudinal Studies Working Paper 11.

Pirus, C. \& Leridon, H. (2010) «Les grandes cohortes d'enfants dans le monde »,Population 65, 4 (671-730).

Pirus C., Bois C., Dufourg M.-N., Lanoë J.-L., Vandentorren S. \& Leridon H. (2010) «La construction d'une cohorte : l'expérience du projet français Elfe »-Population 65, 4 (637-670). Pulkkinen L. \& Kokko K. (2012) «Foundational issues in longitudinal data collection » In: B. Laursen, T. D. Little \& N. A. Card (eds.) (129-147) Handbook of developmental research methods. New York: The Guilford Press.

Ravaud J.-F. (2014) «Les enquêtes en population générale sur le handicap : un outil d'observation essentiel de la statistique publique »- Informations sociales 183, 3 (40-49).

Rayna S. \& Plaisance E. (1997) «L'éducation préscolaire aujourd'hui : réalités, questions et perspectives »- Revue française de pédagogie 119, 1 (107-139).

Special Educational Needs (2001) Code of practice. United Kingdom Education Government. Spira A. (2013) «Les grandes cohortes de santé en France »-Bulletin de l'académie nationale de médecine 2 (315-328). 
Villaume S. \& Legendre E. (2014) « Modes de garde et d'accueil des jeunes enfants en 2013 » - Études et Résultats 896.

Williamson L., Davis E., Priest N. \& Harrison L. (2011) « Australian family day care educators: a snapshot of their qualifications, training and perceived support »-Australasian journal of early childhood 23, 4 (63-68). 